\title{
Avant-propos
}

\section{« La liberté appartient à ceux qui l'ont conquise » André Malraux}

\author{
M. Makaremi ${ }^{1}$, O. Sorel ${ }^{2}$
}

1 Spécialiste qualifié en Orthopédie Dento-Faciale, Pratique libérale, Chargé de recherches en Anthropologie

2 Professeur des Universités, Praticien hospitalier, Responsable du service d'orthodontie, UFR d'Odontologie Rennes

Consacrer un numéro complet de la Revue d'Orthopédie Dento-Faciale aux interactions entre l'anthropologie et l'orthodontie est un choix qui peut de prime abord surprendre le lecteur. Pourtant, il se justifie à bien des égards.

Dans notre organisme, la croissance de la plupart des organes s'effectue de façon efficace et spontanée, sans nécessiter d'intervention thérapeutique. Ce n'est pas le cas de l'appareil manducateur, comme en témoigne la présence endémique de décalage des bases osseuses, de malocclusions ou de dysharmonie dento-maxillaire. Cette réalité clinique est étroitement liée à l'environnement de l'homme anatomiquement moderne : forte réduction des contraintes masticatrices, augmentation des pathologies respiratoires, mélange des populations... Ce numéro questionne sur les raisons de ce déséquilibre auquel s'adresse notre clinique, en ayant recours aux outils et aux apports de la paléopathologie. Une telle exploration donne à l'orthopédie dento-faciale une assise plus profonde, et un rôle plus important que celui de simple correcteur de quelques incisives dystopiques.
Le professeur Philippe l'a parfaitement explicité : nous devons, dans nos diagnostics, nous libérer des normes, afin de voir l'individu au-delà de ses valeurs céphalométriques, d'identifier sa dysmorphose et de développer une réponse thérapeutique qui tend vers l'optimum individuel. Mais disposer d'une telle liberté thérapeutique et de diagnostic a un coût. Si notre perception n'est pas balisée par des valeurs numériques et normatives, nous devons être capables de lire l'architecture cranio-faciale de nos patients et d'intégrer la dysmorphose dans son contexte fonctionnel. L'anthropologie permet d'aborder la complexité de l'architecture cranio-faciale. Traçant l'évolution de la face jusqu'à l'homme anatomiquement moderne, et établissant la genèse des dysmorphoses, elle s'est étoffée de nombreuses études sur la variabilité interet intra-populationnelle. Elle s'impose donc comme un allié précieux pour nous libérer des normes.

Lorsqu'on a pleinement mesuré les causes structurelles des dysmorphoses cranio-faciales chez I'homme, naturellement, on perd beaucoup de sa naïveté au regard de concepts thérapeutiques trop 
simplificateurs. Une connaissance profonde du visage humain, et de la manière dont il s'est construit, développe naturellement notre sens critique quant à nos possibilité thérapeutiques. Cette notion prend une importance d'autant plus marquée que dans le contexte des possibilités offertes par les techniques moderne (minivis, autoligaturant, alliages...), il nous appartient de fixer les limites du mouvement thérapeutique.

L'histoire de notre discipline est liée à l'influence de I'anthropologie dans les concepts thérapeutiques. C'est en observant un crâne sec préhistorique aux arcades alignées et large qu'Angle a eu l'idée de son appareillage Edgewise, qui génère la même expansion sans extraction. De même, l'étude de l'attrition chez les aborigènes est à l'origine du concept de stripping.

C'est à la lumière de toutes ces réflexions qu'a été rédigé ce numéro.

En préambule, un entretien avec le Pr Lieberman, figure éminente de la paléoanthropologie, pose les bases d'une réflexion de fond.

Une étude clinique prospective sur l'influence des contraintes masticatrices fortes dans la stabilisation de l'expansion transversale alvéolaire maxillaire met en lumière l'importance des contraintes masticatrices fortes dans la croissance faciale, ainsi que leur possible utilisation à des fins de thérapeutique orthodontique. Une synthèse bibliographique aborde le facteur ethnique en se penchant sur deux paramètres déci- sifs dans notre matrice d'analyse orthodontique : la maturation dentaire et la position de l'incisive mandibulaire.

L'importance de la prise en compte du facteur ethnique dans la thérapeutique orthodontique est également exposée à travers un exemple clinique.

Le Dr Brondeau se penche sur la complexité de l'architecture cranio-faciale et l'intérêt d'une approche globale du crâne dans l'analyse céphalométrique.

Le Dr Bazert discute de la pertinence d'étendre le regard sur l'individu au-delà de la sphère craniofaciale, à travers une analyse de la posture et des interactions possibles entre les dysmorphoses crano-faciales et l'évolution de notre bipédie.

Enfin, c'est sur l'histoire commune et romanesque de ces deux disciplines, I'anthropologie et I'orthodontie, que revient le Pr Philippe. II explique le besoin que nous avons eu de la craniométrie pour baliser nos mouvements thérapeutiques, et pourquoi nous devons divorcer d'avec cette discipline.

Pour consommer ce divorce, nous devons être maîtres dans notre perception de l'analyse cranio-faciale du patient. Or, cette maîtrise ne s'obtient qu'au prix d'une connaissance de l'architecture cranio-faciale, des causes structurelles des dysmorphoses craniofaciales et d'une connaissance de la variabilité chez I'homme anatomiquement moderne. S'initier à des disciplines fondamentales est ce qu'il nous faut investir pour développer un regard de clinicien libre et performant. 